\title{
Experimental Parametric Identification of a Flexible Beam Using Piezoelectric Sensors and Actuators
}

\author{
Sajad Saraygord Afshari, Hadi Nobahari, and Seyed Ali Hosseini Kordkheili \\ Department of Aerospace Engineering, Sharif University of Technology, Azadi Avenue, P.O. Box 11365-11155, Tehran, Iran \\ Correspondence should be addressed to Hadi Nobahari; nobahari@sharif.edu
}

Received 19 October 2012; Accepted 19 November 2012; Published 29 June 2014

Academic Editor: Abdolreza Ohadi

Copyright (C) 2014 Sajad Saraygord Afshari et al. This is an open access article distributed under the Creative Commons Attribution License, which permits unrestricted use, distribution, and reproduction in any medium, provided the original work is properly cited.

\begin{abstract}
Experimental system identification of a flexible beam based on sweep square excitation is studied. For the purpose of nonparametric identification, an excitation signal is conducted to evaluate the frequency response of the system. The experiment is designed to excite the beam using a piezo actuator, in a way to raise the chance of exciting first three natural modes. In order to find the best linear representation of the real system, two different identification methods are applied. First, autoregressive moving average eXogenous method is employed to identify the transfer function of the beam. Then, the identification is carried out using the subspace identification method to obtain the state space model. A comparison is made between different orders of prediction and the best chosen models of the two identification methods are compared with each other to select the most accurate linear system. Furthermore, as the identified model has to fulfill the controllability and observability conditions, the amenable system is achieved after some order reductions. Two reduction methods called minimum truncation and maximum DC gains matching are utilized to find the most effective reduced order. The outcome of this study will bring in the best linear representation of the beam coupled with piezoelectric sensors/actuators.
\end{abstract}

\section{Introduction}

As the need to lightweight systems increases, we will face more problems on behalf of satisfying inertia and size requirements, and vibration can become a major factor in dynamic behavior of designed structures. This vibration is unwanted and active control methods are required to adapt the dynamic response. Many of the devices used for passive and active structural vibration attenuation are large, heavy, and inappropriate for desired applications. Considering new advances in materials science, which have led to a range of functional materials, we will be able to reach our goals in designing modern configurations with no constraint in dynamical behavior. These structures have been labeled "smart structures" and are commonly used to deal with the vibration control problem in lightweight flexible structures such as flexible beams [1].

Successful vibration control is known as minimizing the possibility of structural failure. In most previous works, various approaches have been utilized to find the theoretical models for the structures embedded with smart materials, such as piezoelectric sensors/actuators. Most of the models were usually limited to either analytical models with ignoring inertia and stiffness of the piezoelectric patches, or finite element models with approximate simulation of the actual identity and properties of the system [1].

Analytical and numerical approaches in modeling dynamical systems coupled with piezo patches are usually time consuming and their applications for accurate control are sometimes defeated by factors such as the assumption of perfect bonding at the interface between the structure and transducers. Besides, these modeling approaches are occasionally impossible for highly complex structures. Considering these limitations, system identification can be introduced as a viable approach in modeling structural systems, such as flexible beams.

In this area, the system identification modeling based on autoregressive moving average eXogenous (ARMAX) and prediction error method (PEM) techniques was successfully developed. The major effort of this study was aimed at establishing a perfect structure model between the system input and output using a parametric system identification 
approach based on nonparametric results. For this purpose, we will apply different methods of linear estimation and the best fitting frequency response will be represented for a flexible beam.

The organization of this paper is as follows. In Section 2, the experimental setup, developed for identification of the fixable beam, is introduced. Parametric and nonparametric system identification and the concept of model reduction are expressed in Section 3. Section 4 is devoted to the experimental results. Finally, a conclusion is made in Section 5.

\section{Experimental Setup}

In order to find an accurate model of the system, a cantilevered aluminum beam $(E=70 \mathrm{GPa}, \nu=0.3, \rho=$ $2700 \mathrm{Kg} / \mathrm{m}^{3}$ ) is developed. Figure 1 shows the experimental setup of the beam in a cantilevered configuration. Two piezoceramic patches are attached on both sides of the beam for the purpose of strain sensing and applying the excitation force to the beam. The aluminum and piezoelectric patches thicknesses are $0.5 \mathrm{~mm}$ and $0.6 \mathrm{~mm}$, respectively. Schematic diagram of the experimental setup is also illustrated in Figure 2.

The beam damping coefficient is very low and it will go under substantial vibrations when excited. Both piezoelectric patches were surface bonded at the cantilever end since this is the best position for sensor and actuator to detect and excite desired modes [2]. The piezo actuator is excited using a PIEZO SYSTEM INC. 20X amplifier, and an ADVANTECH USB-4716 device plays a role as an analog/digital interface.

\section{System Identification}

System identification can be defined as developing a mathematical model for an existing dynamic system and it is widely used in modelling dynamic systems. Capturing the behaviour of a system in a full and accurate model, we will be able to use it to simulate the time response of the system, to develop controller in a fast and precise way using the simulated system behaviour, and so forth [2].

3.1. Nonparametric System Identification. In order to reach an inclusive model of the system, frequency response function (FRF) data is severely required. Hence, an experiment based on sweep square excitation method is performed and the FRF is obtained from a zero initial condition. The input voltage is applied to the piezoceramic actuator of the uncontrolled flexible beam system. The voltage is sensed by the piezo sensor on the other side of the beam [2].

The evaluation test is performed to excite the beam with a square wave voltage, the frequency of which varies from 0.5 to $40 \mathrm{~Hz}$ to have the chance of exciting the first three modes. Time of the experiment is $60 \mathrm{~s}$ with a sampling frequency of $200 \mathrm{~Hz}$ and a digital low pass filter set at $100 \mathrm{~Hz}$. The FRF is calculated using MATLAB signal processing tools.

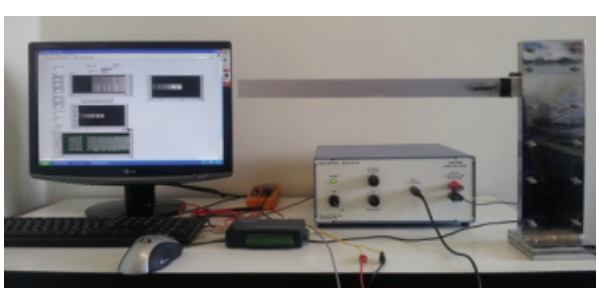

FIGURE 1: Experiment setup of piezo-laminated at smart materials Lab.

3.2. Parametric System Identification. To design a controller for vibration suppression of the first three modes, it is imperative to have a parametric state space model of the system. A proper parametric model can be identified by suitably minimizing the error between the frequency response of predicted and real systems.

In this study, two different system identification methods have been compared to find the most useful and accurate parametric model of the system. First, the well-known method, called ARMAX, is employed to identify the transfer function of the beam structure, equipped with two piezoceramic patches. Then, the identification is carried out using the available subspace identification method of MATLAB platform, namely, pem32, and the state-space model is obtained.

3.3. ARMAX System Identification. Autoregressive moving average models calculate the current system output as a function of past inputs and outputs and ARMAX is an iterative search algorithm which minimizes a quadratic prediction error criterion. The iterations are terminated either when max iteration limit is reached, or when the expected improvement is less than predefined tolerance, or when a lower value of the criterion cannot be found. The general linear form of the ARMAX model is [3]

$$
\begin{aligned}
& y_{t}+a_{2} y_{t-1}+\cdots+a_{n a} y_{t-n a} \\
& =b_{1} u_{t-1}+b_{2} u_{t-2}+\cdots+b_{n b} u_{t-n b},
\end{aligned}
$$

or in discrete linear transfer function form as follows:

$$
\frac{y_{t}}{u_{t}}=\frac{b_{1} z^{-1}+b_{2} z^{-2}+\cdots+b_{n} z^{-n b}}{1+a_{1} z^{-1}+a_{2} z^{-2}+\cdots+a_{n a} z^{-n a}} .
$$

The first practical step in realizing an ARMAX model is to select the degrees of denominator and numerator ( $n a$ and $n b$ ). ARMAX identifies the parameters $a i$ and $b i$ using system input-output data. These data should, if possible, be densely populated in the input space. This will help to reduce the interpolation error when calculating the output for unused data.

3.4. Subspace System Identification. A linear system can be characterized in the state space advanced form as

$$
\begin{gathered}
x(t+1)=A x(t)+B u(t)+K e(t), \\
y(t)=C x(t)+D u(t)+e(t),
\end{gathered}
$$




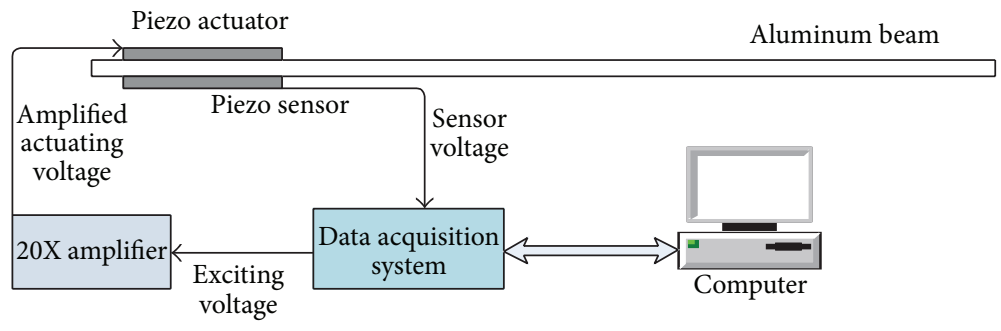

FIGURE 2: Schematic diagram of the Experiment.

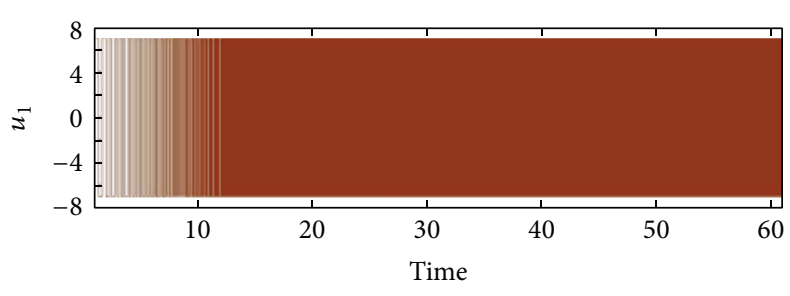

(a)

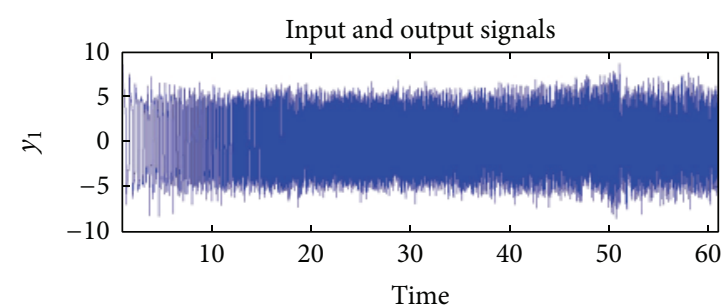

(b)

Figure 3: (a) Excitation signal applied to the system; (b) system experimental time response.

where $e(t)$ is the output that cannot be predicted from the past data, $x(t)$ is the state vector, $y(t)$ is the output, $u(t)$ is the input, and $K$ is the Kalman gain [4].

The subspace method can be used to estimate $A, B, C$, $D$, and $K$ matrices. Assuming that $x(t), y(t)$, and $u(t)$ are known, (3) becomes a linear regression. This will enable us to estimate the matrices $C$ and $D$ by the least-squares method and will lead us to determine $e(t)$. Then, $e(t)$ can be treated as a known signal and this will lead us to determine $A, B$, and $K$ using the least-squares method. The Kalman gain $K$ is computed using Riccati equation. In the above method, it is assumed that $x(t)$ is known; however, it needs to be determined. The states $x(t)$ can be formed as a linear combination of the $k$ step ahead predicted outputs. The above model, derived from the subspace method is then used as the base model for further refining by the prediction error method (PEM). Substantial details for PEM can be found in the MATLAB system identification manual [5]. In this paper, we consider a state space realization that does not model the noise properties; that is, $K=0$.

Here in this study, after applying ARMAX method, MATLAB pem32 tool is utilized to predict the system linear model with PEM method. Comparing the consequences of different prediction orders results in the most effective degrees of freedom for linear estimation of the system using prediction error method and subspace system identification.

3.5. Model Reduction. Finding the most appropriate model of the system is not always enough. Furthermore, the identified model has to fulfill the controllability and observability conditions. Accordingly, after finding the most suitable parametric system, a controllable and observable system can be achieved utilizing some order reduction methods. The order reduction should be performed in a way which results in the slightest deviation from the nonparametric model [6].

Here, the first step after choosing the appropriate linear model is to find the minimal realization of the estimated system. For this purpose, the MATLAB function balreal, is employed. After finding the minimal realization, we may apply modal reduction techniques to reach both controllable and observable system. Again, this objective can be achieved using MATLAB function modred. In both mentioned order reduction functions, two reduction methods, called minimum truncation and maximum DC gains matching, are utilized and the results are compared to find the most helpful reduced order model.

\section{Result and Discussion}

System identification procedures utilized to model the first three modes of vibration in a highly-flexible smart beam. The modes successfully modeled using ARMAX and PEM system identification methods and both obtained models were shown to have sufficient accuracy to design a controller. For each method, different estimation orders have tried to find the best fit model to the original system. Results demonstrated that an order 20 ARMAX and order 25 PEM estimation are applicable in representing desired dynamics of such a cantilevered system. The results show that increasing the system order will not significantly improve the frequency response. Here the models were extracted from the experimental time response of the system under a sweep square excitation as it can be seen in Figure 3.

Finally, a comparison is made between different orders of prediction and the best chosen models of the two identification methods are compared with each other to select the most 

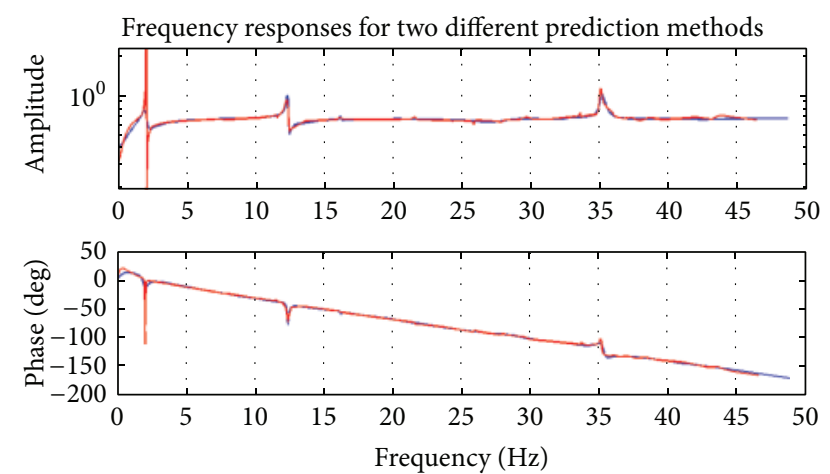

- ARMA prediction-order 20

_ State space prediction with 25 states

FIGURE 4: Frequency responses of estimated parametric models.

accurate representation of the system. The model of order 25, obtained using pem32 method, is finally proposed as it gives a more precise model especially in the first mode frequency. Figure 4 illustrates a comparison between the ARMAX and PEM in their best estimating performance.

Choosing the model of order 25 in state space representation, the observability and controllability of the estimated model are checked, and as it is expected, the model could not satisfy the desired conditions. So MATLAB balreal and modred were utilized consequently in order to reduce the model. Minimal realization of the system which does not affect the frequency response characteristics is obtained by an order 9 system. But, the controllability and observability matrices will not be fully ranked. So, implementing modred, we found a controllable and observable model of order 6 with minimum deviation from the original one.

As it was stated in the previous section, we may use two different methods for reducing the order of system which are matching the DC gains and finding the reduced system using minimum truncation in frequency response. We applied both methods and the results can be seen in Figure 5. In this figure, it can be seen that the parametric system of order 9 matches well the nonparametric model of the original system in its frequency response and it is a good choice for extracting the transfer function. The reduced system of order 6 with minimum truncation method models the first 2 natural frequencies of the system with an acceptable adaptation, although its performance around natural frequencies is not appropriate enough and it cannot model the antiresonance frequencies as well.

The other method, DC gains matching, performs better in the modal frequencies and it is more desirable in active control problems [7]. Finally, step response of the nonparametric identified system is compared with the step responses of different linear models (Figure 6). It can be seen from this figure that the order 6 system using "DC gains matching" method performs better in its time response and it will match the real system after 2 seconds. It is also noticeable that

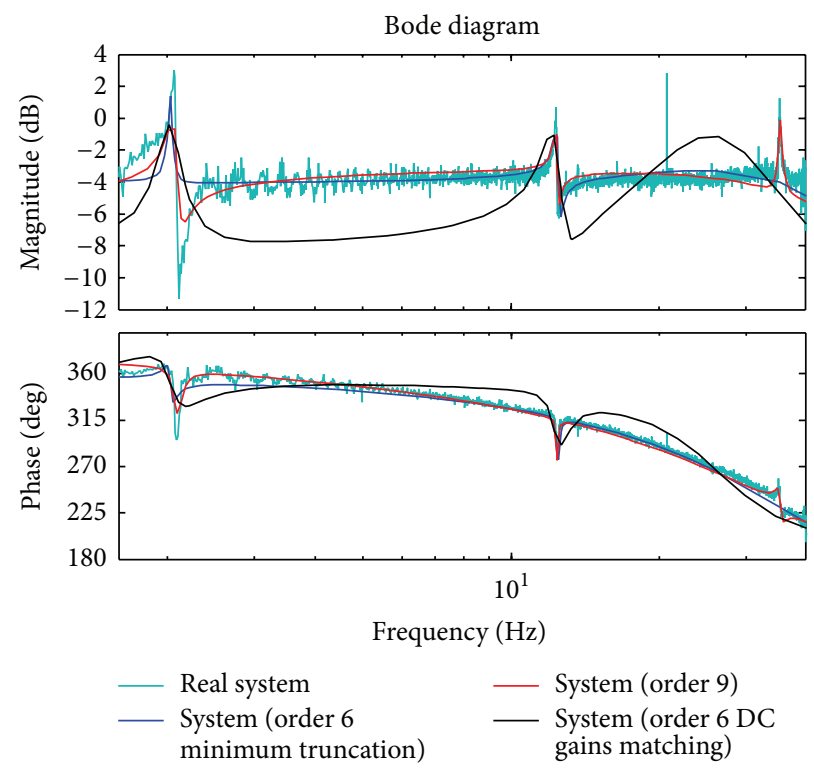

FIGURE 5: Comparison of frequency responses for reduced order models.

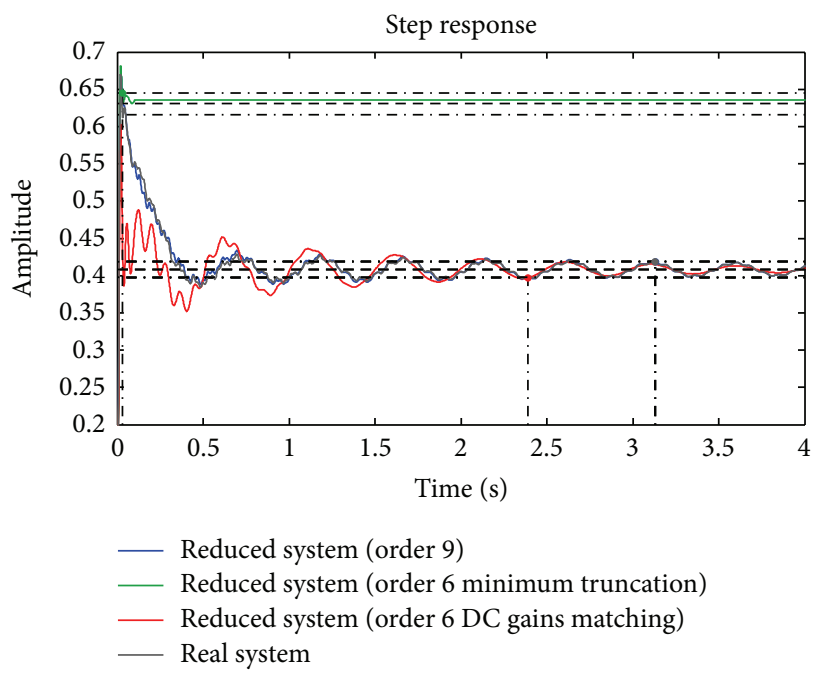

Figure 6: Comparison of step response for different reduced order systems.

the system of order 9 is very similar to the real system in its time response behaviour.

\section{Conclusion}

Problem of identifying a flexible beam coupled with piezoceramic patches is studied in this paper. Parametric system identification procedures are used to obtain a suitable model of the system from its input-output measurement data. Through the comparison of measured and model-based frequency responses, a good representation of the structural dynamics by the identified model was demonstrated and a subspace estimation of order 25 using pem32 was presented as the most effective linear estimation for this system. Finally, by implementing some model reduction methods, an observable and controllable model was achieved in a way, in 
which the system experiences the slightest deviation from the original system. The final outcome of the process provides the system frequency behaviour which can be adopted for different control applications.

\section{Conflict of Interests}

The authors declare that there is no conflict of interests regarding the publication of this paper.

\section{Acknowledgment}

The experiments were conducted at Mechanics of Smart Materials (MSM) Laboratory, Aerospace Engineering Department, Sharif University of Technology.

\section{References}

[1] W. J. Manning, A. R. Plummer, and M. C. Levesley, "Vibration control of a flexible beam with integrated actuators and sensors," Smart Materials and Structures, vol. 9, no. 6, pp. 932-939, 2000.

[2] V. Sethi and G. Song, "Multimodal vibration control of a flexible structure using piezoceramic sensor and actuator," Journal of Intelligent Material Systems and Structures, vol. 19, no. 5, pp. 573582, 2008.

[3] X. Bu, L. Ye, Z. Su, and C. Wang, "Active control of a flexible smart beam using a system identification technique based on ARMAX," Smart Materials and Structures, vol. 12, no. 5, pp. 845850, 2003.

[4] V. Sethi and G. Song, "Optimal vibration control of a model frame structure using piezoceramic sensors and actuators," Journal of Vibration and Control, vol. 11, no. 5, pp. 671-684, 2005.

[5] http://www.mathworks.com/.

[6] Y. Xie, T. Zhao, and G. Cai, "Model reduction and active control for a flexible plate," Acta Mechanica Solida Sinica, vol. 24, no. 5, pp. 467-476, 2011.

[7] X.-J. Dong, G. Meng, and J.-C. Peng, "Vibration control of piezoelectric smart structures based on system identification technique: numerical simulation and experimental study," Journal of Sound and Vibration, vol. 297, no. 3-5, pp. 680-693, 2006. 

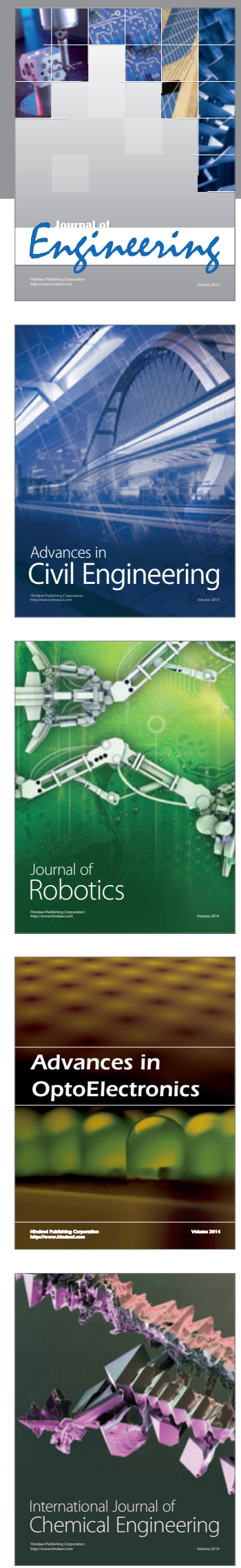

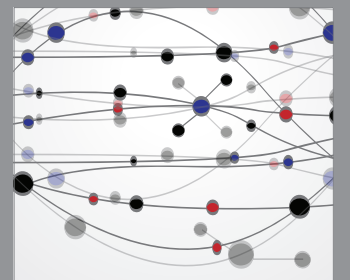

The Scientific World Journal
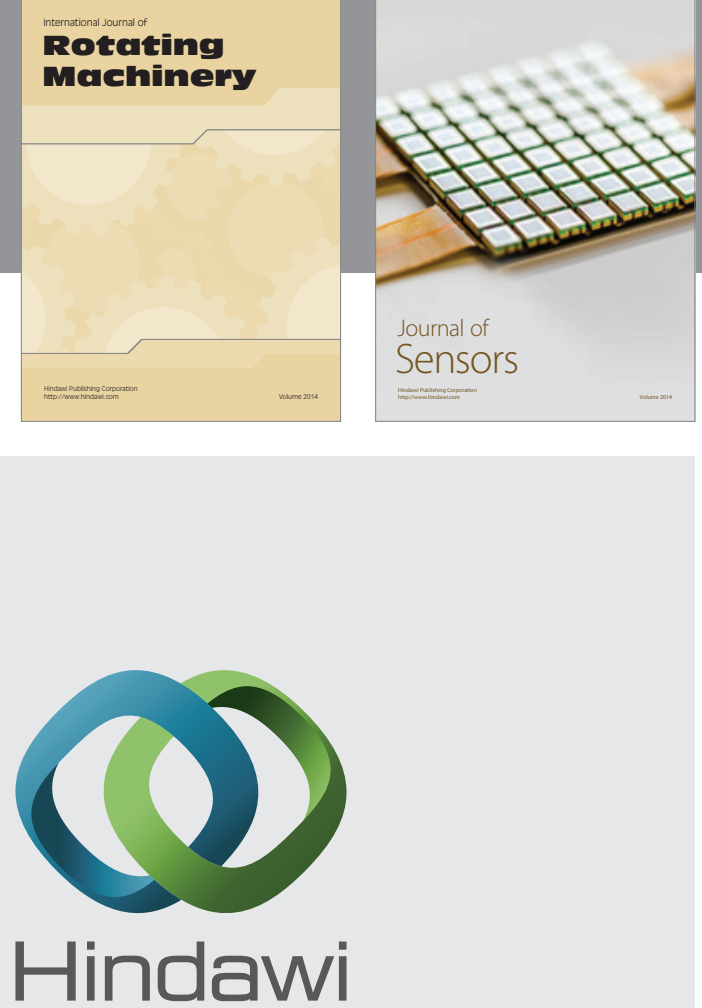

Submit your manuscripts at http://www.hindawi.com
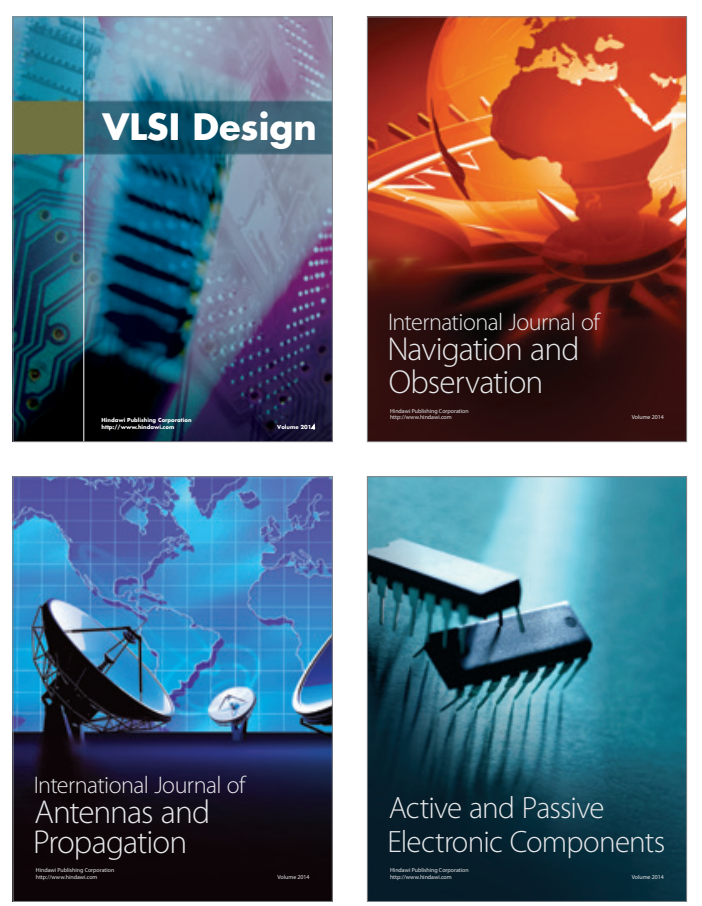
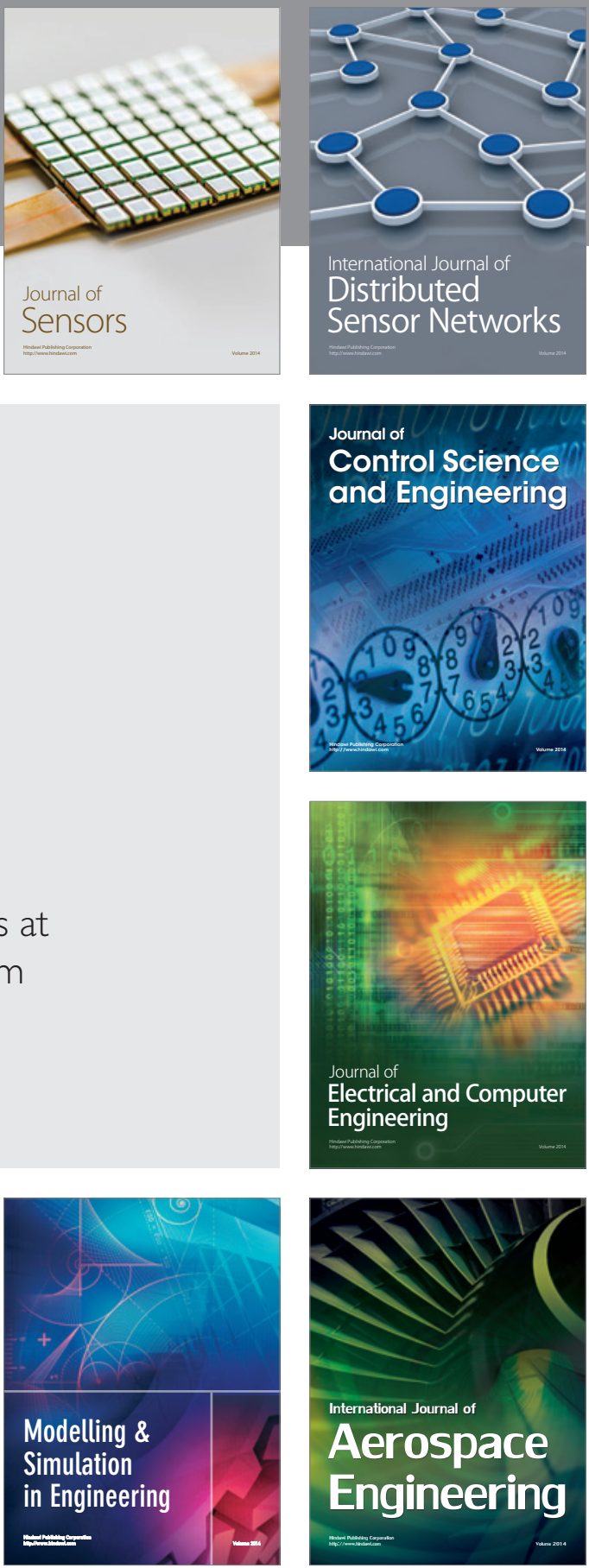

Journal of

Control Science

and Engineering
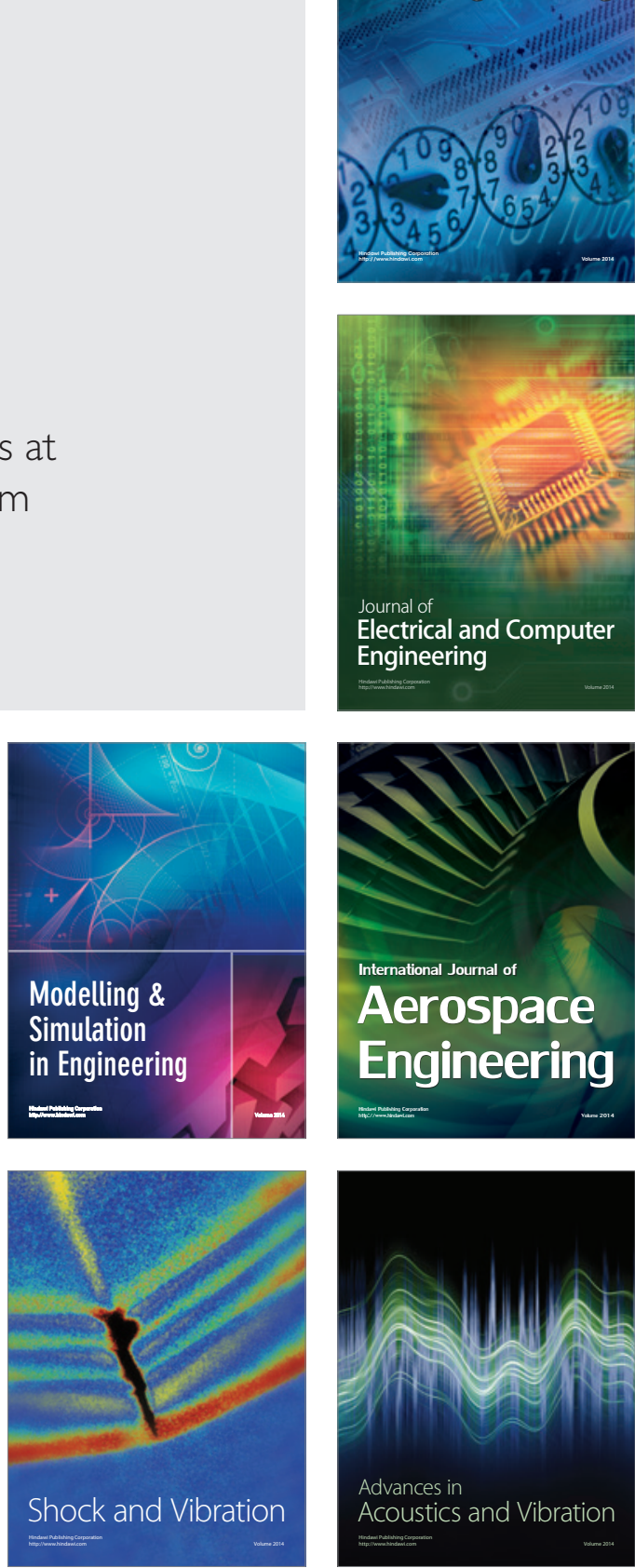